\title{
A Rare Case of Palpebral Cellulitis, a Variation of Pott's Puffy Tumor
}

\author{
Marina Yamamoto ${ }^{\mathrm{a}}$ Nozomu Wakayama ${ }^{\mathrm{b}}$ Yuki Hamajima ${ }^{\mathrm{a}}$ \\ Kohei Miyata $^{a}$ Hiroshi Takahashi $^{c}$ Shinichiro Kobayakawa ${ }^{a}$ \\ aDepartment of Ophthalmology, Nippon Medical School Musashikosugi Hospital,

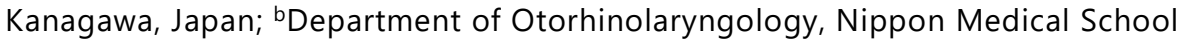 \\ Musashikosugi Hospital, Kanagawa, Japan; 'Department of Ophthalmology, Nippon \\ Medical School, Tokyo, Japan
}

\section{Keywords}

Palpebral cellulitis · Sinusitis · Osteomyelitis · Skin incision · Pott's puffy tumor

\begin{abstract}
A rare case of palpebral cellulitis with simultaneous frontal sinusitis and osteomyelitis is reported. A healthy 45-year-old man presented with left upper eyelid swelling. He was given intravenous meropenem at the local hospital, but he failed to improve. Magnetic resonance imaging showed left frontal and maxillary sinusitis and upper palpebral cellulitis with an abscess. His temperature was $37.6^{\circ} \mathrm{C}, \mathrm{C}$-reactive protein was $1.36 \mathrm{mg} / \mathrm{dL}$, thyroid hormone was elevated, left best-corrected visual activity was 1.2, and intraocular pressure was $25 \mathrm{~mm} \mathrm{Hg}$. He was then given cefazolin intravenously for 3 days but with no improvement. Therefore, the eyelid skin was incised. Postoperatively, the swelling improved significantly. Computed tomography demonstrated osteomyelitis of the left frontal sinus and osteolysis of the inferior wall. This case was considered a variation of Pott's puffy tumor. Bacterial cultures from the cellulitis abscess and sinusitis were negative. As for sinusitis, endoscopic sinusitis surgery (frontal sinus single sinus surgery [Draf III] and Kilian surgery) was performed. During 10 months of followup after the skin incision, no signs of recurrent eyelid swelling were observed.
\end{abstract}




\section{Introduction}

Cellulitis is a common and potentially serious infection caused by bacteria. The bacteria infect the deep layers of skin and subcutaneous tissue. The most common pathogen is Staphylococcus aureus, and other pathogens include Staphylococcus epidermidis, Streptococcus sp., E. coli, Haemophilus influenzae, and a variety of anaerobic bacteria.

Symptoms of cellulitis include erythema, swelling, warmth, pain, tenderness, fever, and formation of blisters and abscesses, etc. Blood tests often show high white blood cell count and C-reactive protein (CRP) levels, but they may not correlate with severity [1]. On computed tomography (CT), high absorption is seen [2], and on magnetic resonance imaging (MRI), low signal intensity is seen on T1-weighted imaging, with high signal intensity on T2-weighted imaging. Therefore, there is no specific blood test or imaging test for cellulitis, which is primarily diagnosed by observation. Complications of cellulitis include necrotizing fasciitis and sepsis. Treatment is oral or intravenous antibiotic therapy, with a cephem type antibiotic or a combination of $\beta$-lactamase inhibitor and penicillin type antibiotic. Causes of palpebral cellulitis are spread from sinusitis, trauma of the frontal bone, surgical history, suppurative dental disease, insect bite, etc. [3].

Pott's puffy tumor (PPT) is a disease that was proposed by Percival Pott in 1768 [4, 5], and it is a rare complication of sinusitis characterized by osteomyelitis of the frontal bone with a subperiosteal abscess presenting as frontal swelling. Incidence of PPT has decreased with the development of antibiotics, but it has not been eradicated [6]. A case of palpebral cellulitis with frontal sinus osteomyelitis caused by spread of a frontal sinusitis, which was considered to be similar to PPT, is reported.

\section{Case Report}

A 45-year-old man who complained of a 1-week history of swelling, erythema, and pain around his left upper eyelid (Fig. 1a-c) was referred to our hospital. The patient had Graves' disease but had discontinued anti-thyroid medications for 1 year. Cellulitis had already been diagnosed and treated with meropenem $0.5 \mathrm{~g} /$ day for 3 days at the local hospital. However, the patient's condition failed to improve.

The patient was already subjected to orbital MRI at the local hospital, which showed left upper palpebral cellulitis, sinusitis in the left frontal and ethmoid sinuses, and no intracranial complications (Fig. 2a-d). The best-corrected visual acuity was 1.2, and the intraocular pressure was $25 \mathrm{~mm} \mathrm{Hg}$ in the left eye. Slit-lamp and fundus examinations of the left eye were normal. On blood tests, CRP was slightly high at $1.36 \mathrm{mg} / \mathrm{dL}$, and the white blood cell count and procalcitonin were within normal limits, $\mathrm{HbA1c}$ was $6.5 \%$, and thyroid hormone was elevated. The patient's temperature was $37.6^{\circ} \mathrm{C}$. The patient was initially given cefazolin $1 \mathrm{~g} /$ day for 3 days but showed no improvement on MRI. Therefore, the skin of the left upper eyelid was successfully incised under local anesthesia, and a large amount of pus and blood was excreted (Fig. 1d-f). Postoperatively, there was significant improvement in the left upper eyelid (Fig. 1g, h). CT examination on the operation day showed left frontal sinus osteomyelitis and osteolysis of the inferior wall of the left frontal sinus connected to the left upper eyelid (Fig. $2 \mathrm{e}-\mathrm{g}$ ). Ceftriaxone $4 \mathrm{~g} /$ day, clindamycin $1.2 \mathrm{~g}$ /day, and fosfomycin $4 \mathrm{~g} /$ day were administered postoperatively. As for sinusitis, endoscopic sinusitis surgery (frontal sinus single sinus surgery [Draf III] and Kilian surgery) was performed. There was no recurrent palpebral cellulitis or sinusitis postoperatively. Bacterial cultures from the cellulitis and sinusitis were negative. 


\section{Discussion}

In the present case, it was thought that the frontal sinus osteomyelitis was caused by frontal sinusitis and that bone destruction at the frontal sinus inferior wall had occurred. Moreover, left upper palpebral cellulitis was the result of spread of the inflammation. The present case was thought to be a variation of PPT because of the osteomyelitis with one or more abscesses outside the bone. However, compared to usual PPT, the abscess was not located anteriorly to the frontal bone, but inferiorly to the upper eyelid. Also, the bone destruction, or the main part of osteomyelitis, involved the inferior wall more than the anterior wall in the present case, and there have been no similar reports to date. As for the bone destruction, it appeared that there was no history of trauma, and as is known, the lower wall was thinner than the frontal wall.

The major symptoms of PPT are frontal swelling, headache, fever, and nasal discharge. Except for the frontal swelling, these symptoms are nonspecific. It may be difficult to suspect frontal sinus osteomyelitis until frontal swelling becomes evident. However, when the inflammation of PPT diffuses, it can cause pachymeningitis, an epidural or subdural abscess, and brain abscess, which would then require surgical drainage [7-9]. Thus, PPT is a risk factor of intracranial infection, and it has been reported that $85 \%$ of PPT cases had intracranial complications [9]. PPT used to have a poor prognosis but is currently curable due to the advent of antibiotic therapy [9].

The oxygen concentration is lower in the frontal sinus than in other sinuses. This is the reason why many reports found anaerobic bacteria, such as Fusobacterium sp. or Bacteroides sp., as pathogens for PPT [7, 9]. There was no causative organism identified in the present case, which was considered to be the effect of the antibiotic administered at the local hospital. Cefazolin was used initially in our department; the first-generation cephem antibiotics are better against gram-positive bacteria, but they are weak against anaerobic bacteria. A broadspectrum antibiotic approach, like a combination of ceftriaxone and clindamycin, should be chosen for anaerobic bacteria. The skin incision of the left upper eyelid was performed because of subcutaneous abscess formation, after which the swelling improved significantly. Severe complications, such as necrotizing fasciitis, sepsis, and intracranial lesions, could be prevented with an early surgical response.

It is necessary to keep in mind that sinusitis can cause palpebral cellulitis, orbital cellulitis, and osteomyelitis. Also, the etiology of cellulitis must generally be established, by clinical or imaging means, so as to exclude an autoimmune or malignant cause, or to determine the boundaries of the inflammatory process or find a foreign body. This requires a multidisciplinary approach with the cooperation of the ophthalmologist, the radiologist, and the otolaryngologist. In cellulitis resistant to initial treatment, CT and MRI should be performed early to prevent missing the opportunity for diagnosis and treatment. Additionally, in resistant cases of preseptal cellulitis in adults, an antibiotic effective against anaerobic bacteria should be added to the treatment regimen.

\section{Statement of Ethics}

Written informed consent was obtained from the patient for publication of this case report and any accompanying images. The authors have no ethical conflicts to disclose. 
Disclosure Statement

The authors declare that there are no conflicts of interest regarding the publication of this paper.

\section{Funding Sources}

There were no funding sources.

\section{Author Contributions}

M.Y., N.W., H.T., and S.K. participated in drafting the manuscript. M.Y., N.W., Y.H., K.M., and S.K. participated in diagnosis and treatment of the patient.

\section{References}

1 Sakurai H. Cellulitis. Jpn J Pediatr. 2018 Dec;71(12):2533-6.

2 Inada N. Orbital cellulitis. Jpn J Clinic Ophthalmol. 2016 Oct;70(11):141-6.

3 Ferguson MP, McNab AA. Current treatment and outcome in orbital cellulitis. Aust N Z J Ophthalmol. 1999 Dec;27(6):375-9.

4 Guillén A, Brell M, Cardona E, Claramunt E, Costa JM. Pott's puffy tumour: still not an eradicated entity. Childs Nerv Syst. 2001 May;17(6):359-62.

5 Clark JR, Lim JK, Poole M. Pott's puffy tumour: a clinical variant. Aust N Z J Surg. 1999 Oct;69(10):759-62.

6 Bağdatoğlu C, Güleryüz A, Ersöz G, Talas DU, Kandemir O, Köksel T. A rare clinical entity: pott's puffy tumor. A case report. Pediatr Neurosurg. 2001 Mar;34(3):156-8.

7 Gupta M, El-Hakim H, Bhargava R, Mehta V. Pott's puffy tumour in a pre-adolescent child: the youngest reported in the post-antibiotic era. Int J Pediatr Otorhinolaryngol. 2004 Mar;68(3):373-8.

8 Pender ES. Pott's puffy tumor: a complication of frontal sinusitis. Pediatr Emerg Care. 1990 Dec;6(4):280-4.

9 Bambakidis NC, Cohen AR. Intracranial complications of frontal sinusitis in children: pott's puffy tumor revisited. Pediatr Neurosurg. 2001 Aug;35(2):82-9. 


\section{Case Reports in Ophthalmology}

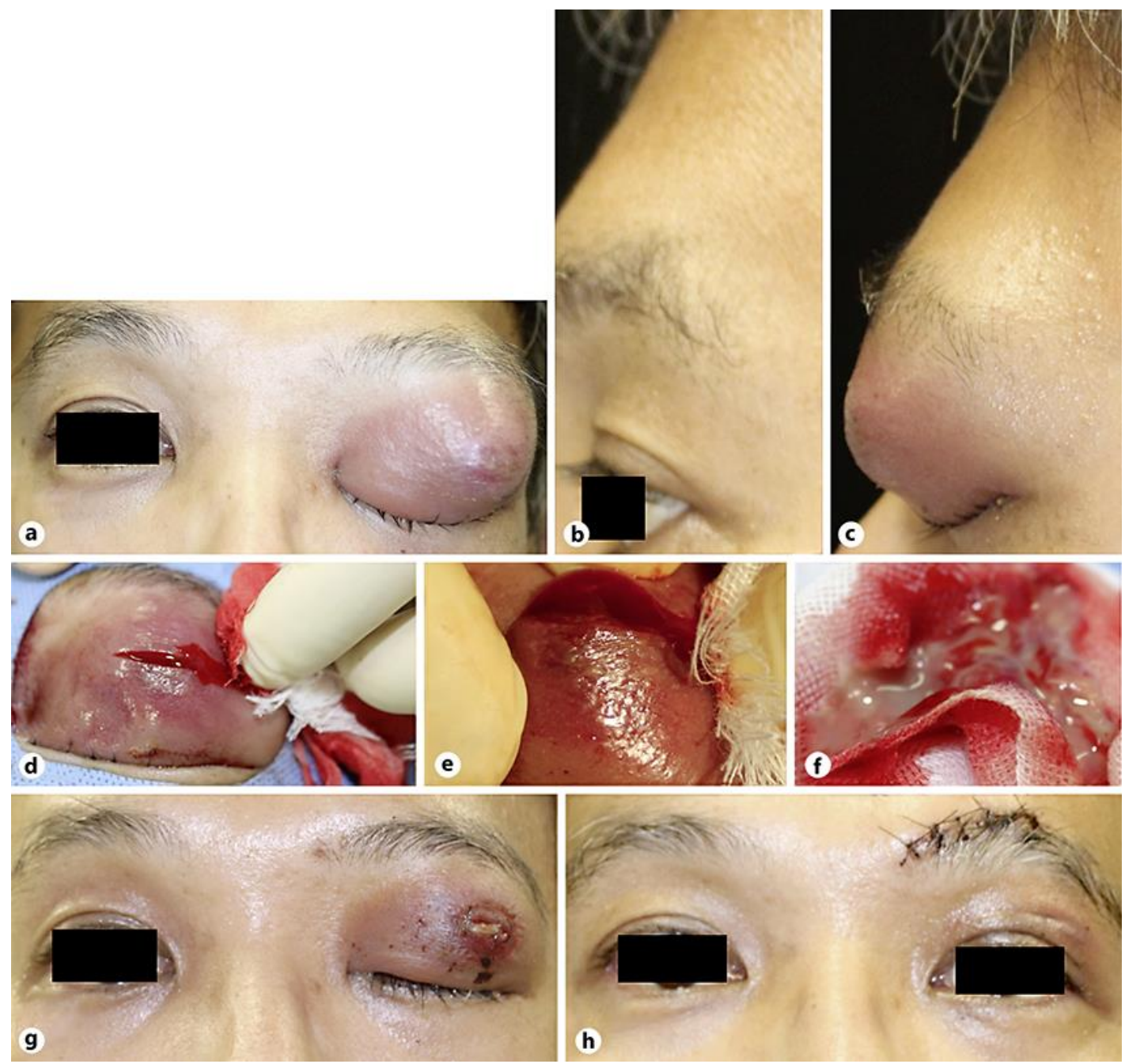

Fig. 1. a-c Preoperative photograph of the face. a Frontal view. $\mathbf{b}$ Right eye (mirror-reversed image). $\mathbf{c}$ Left eye. $\mathbf{d}-\mathbf{f}$ Intraoperative findings. $\mathbf{d}$ Horizontal skin incision. $\mathbf{e}$ The skin is pressed by the fingers. $\mathbf{f}$ A large amount of pus drains out. $\mathbf{g}$, $\mathbf{h}$ Photographs of the face on the 1st (g) and 20th (h) postoperative days. The left palpebral swelling has improved significantly. 


\section{Case Reports in Ophthalmology}
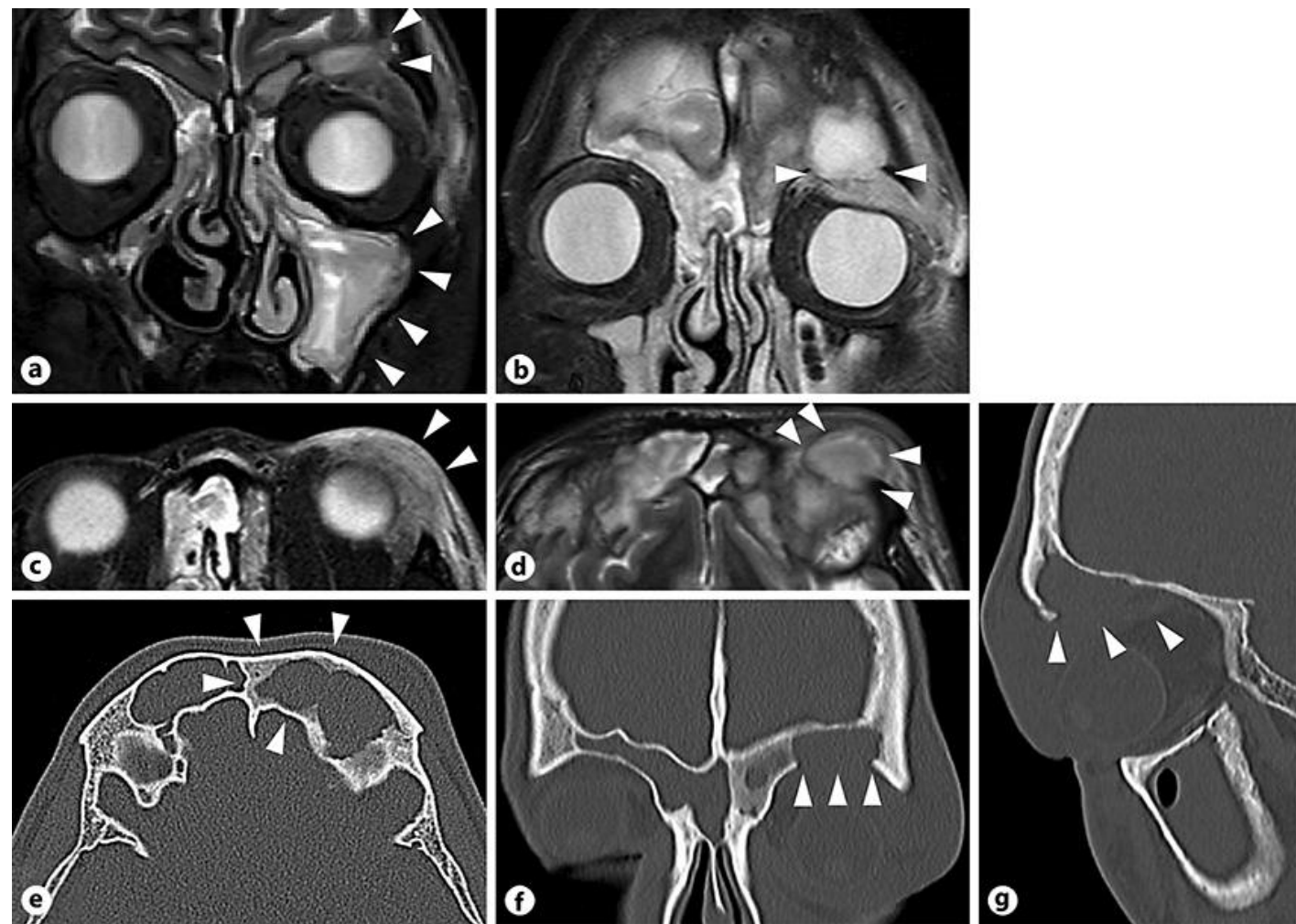

Fig. 2. a-d Initial orbital MRI. a, b Fat-suppressed T2-weighted coronal image. a Sinusitis centering on the left frontal and maxillary sinuses (arrowheads). b Left frontal sinusitis connected to the palpebral abscess (arrowheads). c Fat-suppressed T2-weighted axial image shows left palpebral cellulitis (arrowheads). d T2-weighted axial image shows left palpebral abscess (arrowheads). e-g Orbital CT scan after the eyelid operation. e Axial image shows osteomyelitis of the left frontal sinus anterior, inferior, and posterior walls (arrowheads). Coronal image (f) and sagittal image (g) show bone destruction of the left frontal sinus inferior wall (arrowheads). 